\begin{tabular}{|c|c|}
\hline Title & A mplified spontaneous emission from a surface modified GaN film fabricated under pulsed intense UV laser irradiation \\
\hline Author(s) & Fujiwara, Hideki; Sasaki, Keiji \\
\hline Citation & $\begin{array}{l}\text { A pplied Phy sics Letters, 113(17), } 171606 \\
\text { https://doi.org/10.1063/1.5040551 }\end{array}$ \\
\hline Issue Date & $2018-10-22$ \\
\hline Doc URL & http://hdl.handle.net/2115/75819 \\
\hline Rights & $\begin{array}{l}\text { This article may be downloaded for personal use only. A ny other use requires prior permission of the author and AIP } \\
\text { Publishing.The following article appeared in Hideki Fujiwara and Keiji Sasaki. A mplified spontaneous emission from a } \\
\text { surface modified GaN film fabricated under pul sed intense UV Iaser irradiation A ppl. Phys. Lett. 113, 171606 (2018) } \\
\text { and may be found at https://ai p.scitation.org/doi/10.1063/1.5040551. }\end{array}$ \\
\hline Tyре & article \\
\hline File Information & GaN_Random_laser.pdf \\
\hline
\end{tabular}

Instructions for use 
Amplified spontaneous emission from a surface-modified GaN film fabricated under pulsed intense UV laser irradiation

Hideki Fujiwara, and Keiji Sasaki

Citation: Appl. Phys. Lett. 113, 171606 (2018); doi: 10.1063/1.5040551

View online: https://doi.org/10.1063/1.5040551

View Table of Contents: http://aip.scitation.org/toc/apl/113/17

Published by the American Institute of Physics

\section{Lake Shore}

CRYOTRONICS

Sensors, Controllers, Monitors

from the world leader in cryogenic thermometry 


\title{
Amplified spontaneous emission from a surface-modified GaN film fabricated under pulsed intense UV laser irradiation
}

\author{
Hideki Fujiwara ${ }^{a)}$ and Keiji Sasaki \\ Research Institute for Electronic Science, Hokkaido University, Sapporo 001-0020, Japan
}

(Received 18 May 2018; accepted 12 October 2018; published online 26 October 2018)

\begin{abstract}
We propose a simple method for fabricating random structures directly on Mg-doped GaN thin films. The process is relatively simple, involving only irradiation with strong UV pulses from a fabrication laser on the thin-film surface. After intense UV laser pulses $\left(>400 \mathrm{MW} / \mathrm{cm}^{2}\right)$ are irradiated on the flat GaN film, the surface is roughened and quasi-periodic structures form. When the roughened surface is excited with laser light of intensity about 10 times smaller than the fabrication laser intensity, emission increases around $367 \mathrm{~nm}$, and spectral narrowing and threshold behavior are observed. Because such behaviors are not observed without the application of intense laser irradiation, we conclude that amplified spontaneous emission is induced in the modified GaN surface by the intense UV laser pulse irradiation. This method offers the possibility of easy and direct fabrication of microscale random-laser devices on semiconductor substrates. Published by AIP Publishing. https://doi.org/10.1063/1.5040551
\end{abstract}

Gallium nitride $(\mathrm{GaN})$ is an attractive material for various semiconductor applications because of its high emission efficiency, high electrical conductivity, high binding energy, transparency to visible light, and its wide emission range. ${ }^{1-3}$ GaN has been widely applied in UV-blue light emitting diodes (LEDs) for use in sterilization, optical recording, and laser displays. These GaN-based LEDs typically feature a Fabry-Perot cavity structure formed from the end facets of a cleaved or plasma-etched GaN film grown on a hard substrate. ${ }^{4,5}$ However, due to the hardness of the substrate, the cleaving process remains a manufacturing challenge in terms of complexity and consistency. Furthermore, although micro-pillars, nano-columns, and micro-disks have also been adopted as laser-cavity structures and exhibit highperformance laser characteristics, ${ }^{6-8}$ these also require complex fabrication processes with high fabrication costs. In contrast, random structures can be produced with relative ease and low cost. These structures are typically composed of nanoparticle assemblies or surface roughness, and are also enabled to induce laser oscillations due to random optical feedback in the structures caused by multiple light scattering, so they are referred to as random lasers. ${ }^{9-12}$ These random lasers have recently been studied as unique speckle-free laser light sources that are particularly well-suited for use in sensors and full-field imaging. ${ }^{12}$ Thus, such a random structure could be one of the promising structures for the fabrication of GaN-based laser and/or LED sources; ${ }^{13,14}$ e.g., Zhu et al. proposed a simple and low-cost GaN-film laser, in which random lasing in GaN epitaxial films is induced by weak coherent feedback that is caused by randomly distributed defect pits acting as scatterers.

In our previous studies, ${ }^{15-18}$ we proposed fabrication methods for random lasers that allow control of the lasing modes by selecting the size of spherical nanoparticles or nanorod arrays. These methods were intended to produce random lasers with a low excitation threshold. Considering

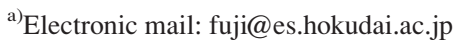

the potential applications such as LEDs and photovoltaic devices, improving the electrical-drive properties and lowering the excitation threshold of random lasers and LEDs are crucially important. The cost and complexity of the method for directly fabricating nanostructures on semiconductor substrates also need to be reduced. As an approach to fabricating structures on a substrate, we have paid attention to the fabrication technique for laser-induced periodic structures, ${ }^{19-22}$ in which clear periodic surface structures can be fabricated on various material surfaces, such as glasses, metals, and semiconductors, directly and simply with irradiation by a pulsed intense linearly polarized laser. This method offers the advantages of direct fabrication on the material surface, which is advantageous for the electric-drive properties of the lasing device. In addition, the laser-induced periodic structures offer the chance to control the structure by adjusting the fabrication conditions, resulting in lowering thresholds.

In this study, we propose a simple and low-cost method for fabricating a GaN-based light emitting device, by which a steep increase in UV emission was observed after only irradiating a GaN film with a strong laser to roughen the surface. This technique may be applicable to produce GaN-film lasers at a very low cost. In addition, the results have important implications for fabrication processes with wider-bandgap materials such as BN and diamond, as a similar method may be used to fabricate short-wavelength ultraviolet random lasers from these materials.

In the experiments, a commercially available $\mathrm{Mg}$-doped GaN film (4- $\mu \mathrm{m}$ thickness on a sapphire substrate, MTK Co., Ltd.) was placed on a microscope stage. To fabricate the roughened GaN surface, intense UV laser pulses $(355 \mathrm{~nm}$, $1 \mathrm{kHz}, 300 \mathrm{ps}$, spot diameter $\sim 50 \mu \mathrm{m}$, linearly polarized) were irradiated through an objective lens $(60 \times, \mathrm{NA}=0.85)$. When the irradiated intensity was above $\sim 400 \mathrm{MW} / \mathrm{cm}^{2}$, we observed structures fabricated on the surface [Fig. 1(a)]. Figures 1(b) and 1(c) show SEM images of the GaN surface after the irradiation of intense pulses (intensity: $\sim 550 \mathrm{MW} /$ $\mathrm{cm}^{2}$, irradiation time: $10 \mathrm{~s}$ ). The polarization is indicated by 

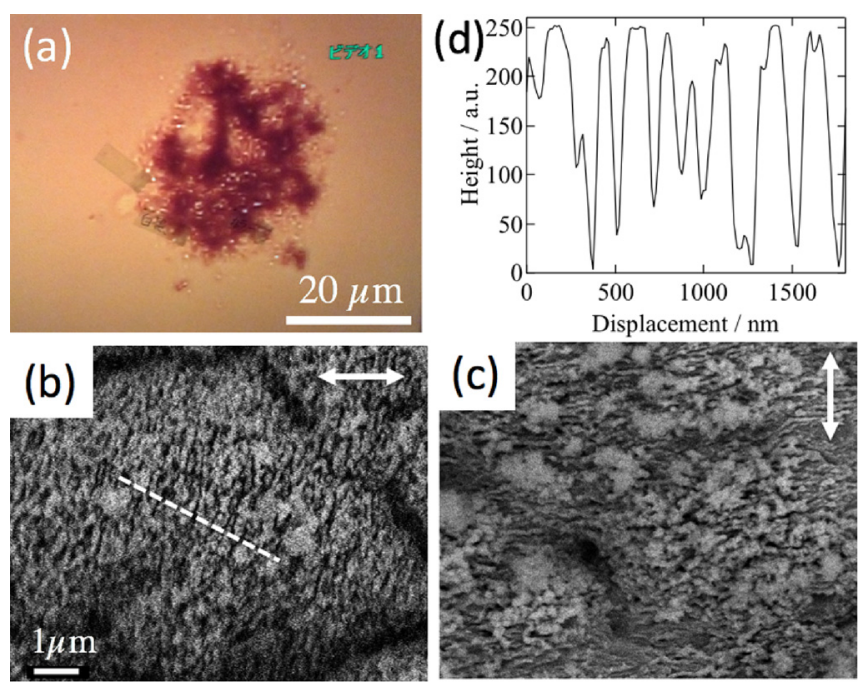

FIG. 1. GaN surfaces after surface treatment with intense UV laser irradiation pulses. (a) Microscopy image. (b) and (c) SEM images of the GaN surface after intense laser irradiation with different polarizations, indicated by arrows. Irradiated UV laser intensity and time in all cases were $\sim 500 \mathrm{MW} /$ $\mathrm{cm}^{2}$ and $10 \mathrm{~s}$. (d) Line profile along the white dashed line marked in (b).

the arrows in the figure. From the results, we found that the smooth surface of the GaN film was roughened by the intense irradiation pulses. Quasi-periodic structures with a periodicity of $250-300 \mathrm{~nm}$ were confirmed [Fig. 1(d)], and they were formed perpendicular to the polarization direction. Similar results have been reported with the use of intense femtosecond-laser irradiation to produce laser-induced periodic structures. ${ }^{19-22}$ After fabricating the modified GaN surfaces with intense pulses, we measured the emission spectra of the samples using the same laser at an intensity of $100 \mathrm{MW} / \mathrm{cm}^{2}$ as an excitation source. Hereafter, we refer to the UV pulsed laser used for surface modification as a fabrication laser and when used for emission spectral measurements, we refer to it as an excitation laser. Note that we attempted to confirm the lasing using an Mg-doped (p-type) $\mathrm{GaN}$ in the UV region (near-band-edge emission) because lasing oscillation using non-doped (n-type) GaN had been reported in various kinds of resonators. Because $\mathrm{Mg}$ ions generate other radiative transitions $(450 \mathrm{~nm})$, there is a possibility that it may prevent lasing at the near-band-edge.

Figure 2 shows the broadband emission spectra of the GaN films before and after surface modification, as induced by weak excitation. The laser intensity during the surface modification process was fixed at $\sim 550 \mathrm{MW} / \mathrm{cm}^{2}$, which is the same intensity that was used to fabricate the samples shown in Fig. 1. Figure 2(a) shows the emission spectra from the untreated sample with excitation intensity $0.13-18.2 \mathrm{MW} / \mathrm{cm}^{2}$. A broad peak appears around $450 \mathrm{~nm}$, which is typically attributed to doped $\mathrm{Mg}$ ions. ${ }^{23}$ If the excitation intensity is increased, the near-band-edge emissions at $\sim 370 \mathrm{~nm}$ and visible emissions around 550-600 nm increase. Comparing this result with the emission spectra after surface modification with intense UV irradiation pulses, slight changes can be observed in Fig. 2(b). The emission intensity at $450 \mathrm{~nm}$ slightly decreases and the intensities of the UV and visible emissions slightly increase. These changes occur because the evaporation of $\mathrm{Mg}$ ions increases UV emissions,

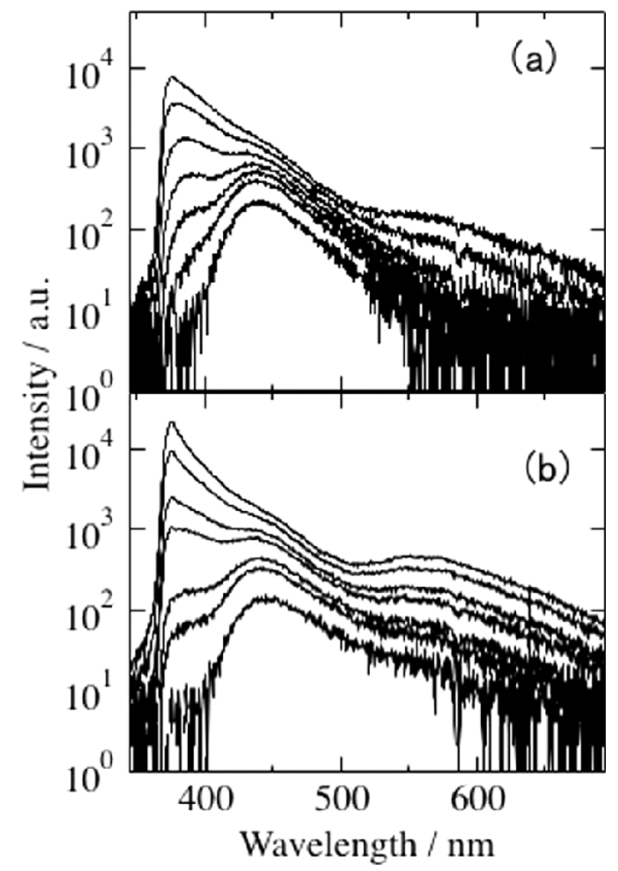

FIG. 2. Emission spectra (a) before and (b) after intense pulsed UV laser irradiation $\left(550 \mathrm{MW} / \mathrm{cm}^{2}\right.$ and $\left.10 \mathrm{~s}\right)$ under weak excitation. Excitation intensities were $0.13,0.55,1.1,2.7,5.3,12.0$, and $18.2 \mathrm{MW} / \mathrm{cm}^{2}$, respectively, which are much smaller than the thresholds discussed below.

and the increase in structural defects because of the strong laser irradiation also increases visible emissions.

We measured the dependence of the emission spectra on the excitation intensity to confirm emission behaviors induced by the surface-modified GaN film. Figure 3 plots the integrated emission intensities and emission spectra versus excitation intensity. The excitation intensities were varied from 9 to $225 \mathrm{MW} / \mathrm{cm}^{2}$, which were sufficiently lower than

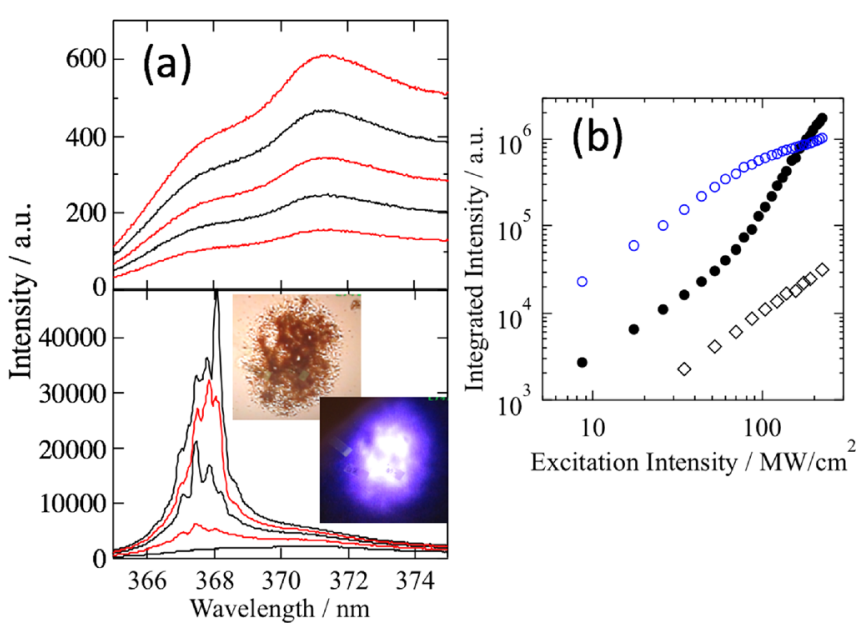

FIG. 3. (a) Emission spectra from a non-treated and modified GaN surfaces (upper and lower panels, respectively) under strong excitation. The excitation intensities are 52, 121, 156, 190, and $225 \mathrm{MW} / \mathrm{cm}^{2}$ from bottom to top. The insets show the modified surface and its emission images. (b) Integrated emission intensities versus excitation intensity. Solid circles: wavelengths from 366 to $369 \mathrm{~nm}$, open circles: wavelengths from 371 to $385 \mathrm{~nm}$ emitted from the modified GaN surface. Diamonds: wavelengths from 366 to $369 \mathrm{~nm}$ for the non-treated flat GaN surface. Note that the maximum excitation intensity is still about 3 times smaller than that used for the fabrication of surface-modified GaN surfaces and no damage was caused to the flat GaN surface by the emission-measurement procedure. 
the fabrication laser intensity $\left(\sim 560 \mathrm{MW} / \mathrm{cm}^{2}\right)$, but much higher than those used for the results shown in Fig. 2. For comparison, first, we measured the emission spectra from a flat $\mathrm{GaN}$ surface (before surface modification) and found only broad and weak emissions around $368 \mathrm{~nm}$, which is the near-band-edge emission from GaN. However, no spectral change and threshold behavior was observed in this range of excitation intensity [shown in the upper panel in Fig. 3(a) and marked with diamonds in Fig. 3(b)]. However, after surface modification, the emission spectrum measured at the same position indicated an increase in UV emission around $368 \mathrm{~nm}$ with narrowing spectral shape [lower panel in Fig. 3(a)]. The insets in Fig. 3(a) show the modified surface and its emission images. We found that strong UV emission was induced from the modified region (black region in the left image) and threshold behaviors could be observed from these bright spots, while from untreated surfaces, we only observed weak spontaneous emission. These results imply that the threshold behavior is strongly related to the surface modification. Figure 3(b) plots emission intensities against excitation intensity. Emission intensities integrated around the broad emission peak from 371 to $385 \mathrm{~nm}$ (open circles) show linear growth until saturation, while the intensities integrated from 366 to $369 \mathrm{~nm}$ (solid circles) exhibit a steep increase at the excitation intensity of $\sim 70 \mathrm{MW} / \mathrm{cm}^{2}$, which is about 8 times smaller than the fabrication laser intensity. However, from the flat GaN surface, the UV emission intensity is relatively small and increases linearly with increasing excitation intensity. Note that we observed no damage to the surface caused by the excitation measurements of the flat surface. Also note that similar behaviors could be observed from the undoped $\mathrm{GaN}$ thin film only after the intense fabrication laser irradiation. Although the threshold behaviors were observed, peak fluctuations that have generally been observed in random lasers could not be confirmed when we changed the excitation intensity. We note that it may suggest the possibility of amplified spontaneous emission rather than the possibility of random lasing with coherent random feedback. On the other hand, in the experiments, such threshold behavior and increase in emission intensity could not be confirmed from a flat GaN substrate and observation was carried out in the direction perpendicular to the substrate with the small excitation spot diameter of $50 \mu \mathrm{m}$. Therefore, we considered that, due to the surface modification, the observed phenomena could be induced by the increase in the optical path lengths of excitation light or emission due to the surface modification.

To investigate the influence of surface modifications, we measured the emission spectra and took SEM images of modified $\mathrm{Mg}$-doped $\mathrm{GaN}$ surfaces with different irradiation intensities. Figure 4(a) shows the emission spectra from $\mathrm{GaN}$ surfaces fabricated with fabrication laser intensities from 347 to $644 \mathrm{MW} / \mathrm{cm}^{2}$ and a fixed irradiation time $(10 \mathrm{~s})$. The excitation laser intensity for these measurements was fixed at $248 \mathrm{MW} / \mathrm{cm}^{2}$, which was sufficiently higher than the observed thresholds shown in Fig. 3. If the fabrication laser intensity was relatively weak, less than $\sim 500 \mathrm{MW} / \mathrm{cm}^{2}$, only a broad emission peak was observed even though the UV emission increased as the fabrication laser intensity increased. When the fabrication laser intensity was $\sim 550 \mathrm{MW} / \mathrm{cm}^{2}$, the shoulder of the broad emission spectrum

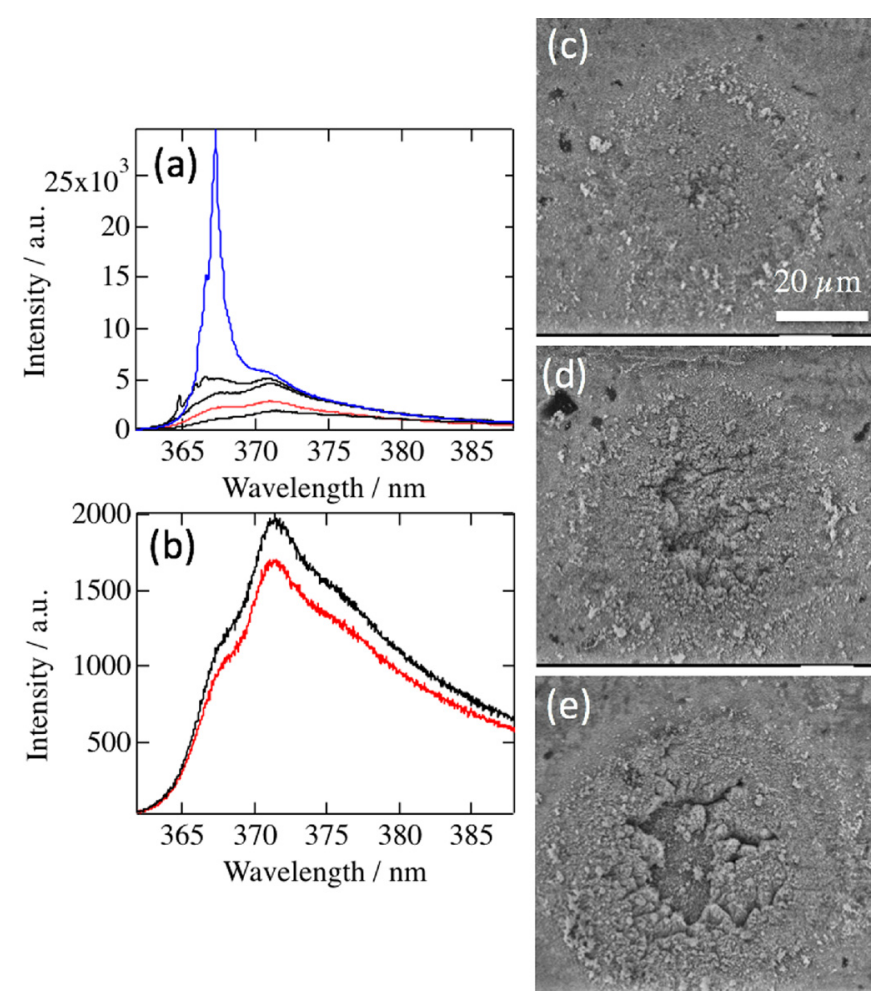

FIG. 4. Emission spectra from the modified GaN surfaces with different UV pulse irradiation conditions. (a) Fabrication irradiation intensities were 347, $495,545,594$, and $644 \mathrm{MW} / \mathrm{cm}^{2}$, while the irradiation time was fixed at $10 \mathrm{~s}$. The excitation intensity for measuring emission spectra was fixed at $248 \mathrm{MW} / \mathrm{cm}^{2}$, which is sufficiently higher than typical threshold values in Fig. 3. The blue and red lines highlight the results from samples made at irradiation intensities of 594 and $644 \mathrm{MW} / \mathrm{cm}^{2}$, which correspond to the SEM images in (d) and (e). (b) The black and red lines plot the emission spectra of samples fabricated with different irradiation times (black line: $10 \mathrm{~s}$, red line: $2 \mathrm{~min}$.) and fixed fabrication laser intensity at $495 \mathrm{MW} / \mathrm{cm}^{2}$. (c-e) SEM images of the samples made with fabrication laser intensities of 347,594 , and $644 \mathrm{MW} / \mathrm{cm}^{2}$.

grew around $367 \mathrm{~nm}$. When the fabrication laser intensity was increased to $\sim 600 \mathrm{MW} / \mathrm{cm}^{2}$, a steep increase in the emission intensity and spectral narrowing at $367 \mathrm{~nm}$ were clearly observed [blue line in Fig. 4(a)]. This result is marked with a blue line in the figure. However, further increases of the fabrication laser intensity led to a sudden decrease in UV emission that only showed a broad emission, the result of which is marked with a red line in the figure. These phenomena were observed repeatedly at other positions on the modified GaN surface. In addition, Fig. 4(b) indicates the emission spectra of the samples prepared with different irradiation times. The fabrication laser intensity was set at $495 \mathrm{MW} / \mathrm{cm}^{2}$, which is slightly lower than the fabrication laser intensity that we could observe from the threshold behavior of the samples, and the irradiation time was set to be either $10 \mathrm{~s}$ (black line) or $2 \mathrm{~min}$ (red line). Even with the longer irradiation time, only broad emissions were observed. This means that irradiation intensity is the dominant factor in fabricating surface structures that initiate the observed phenomena.

Figures 4(c)-4(e) show SEM images of the samples used in Fig. 4(a), for which the fabrication laser intensities were (c) 347 , (d) 545 , and (e) $644 \mathrm{MW} / \mathrm{cm}^{2}$, respectively. From these SEM images, we find that increasing the fabrication laser intensity increased the surface roughness. 
However, when the fabrication laser intensity was too strong [644 MW/ $/ \mathrm{cm}^{2}$, marked with the red line in Fig. 4(a)], the irradiating laser ablated the GaN thin film so that the sapphire substrate is visible from the surface [Fig. 4(e)]. From the above results, we conclude that, with weak fabrication intensities, the surface is not modified enough to provide multiple light scattering and, at stronger fabrication intensities, the thin GaN layer is nearly ablated under the irradiation spot so that insufficient amplification is produced. Thus, the optimal irradiation intensity for fabricating the surface roughness exists. In this study, to fabricate a modified GaN surface that undergoes multiple light scattering, we found that the optimal irradiation conditions were $\sim 550 \mathrm{MW} / \mathrm{cm}^{2}$, samples of which are shown in Fig. 3.

As quasi-periodic structures on the GaN surface were also fabricated by intense fabrication laser irradiation [see Figs. 1(b) and 1(c)], this periodicity may have been responsible for lasing feedback. However, the emission spectral measurements revealed no clear influence of the periodic structures (different peak wavelengths were observed at different positions). In addition, although we tested the detection polarization dependence, we also observed no clear difference with different polarizations. Thus, in contrast with the previous studies of distinct periodic structures fabricated by femtosecond-pulsed laser irradiation, ${ }^{19-22}$ our experimental results could be explained as an effect of multiple light scattering within the modified surface, rather as an effect of periodic structures. This effect would be explained by the low profile quality of the picosecond-pulsed laser, which is evidenced by the modified surface shown in Fig. 1. Our findings may suggest the possibility of a simple method to directly fabricate LEDs or distributed-feedback lasers on a GaN surface at a greatly reduced cost, if we use a femtosecond laser with a good profile, as used in previously reported studies. $^{19-22}$

In summary, we demonstrated the fabrication of UV light emitting devices from GaN thin films in a process that uses only strong UV laser pulses $\left(\sim 550 \mathrm{MW} / \mathrm{cm}^{2}\right)$. After irradiating intense UV pulses on the GaN thin film, the surface was roughened and near-band-edge emissions increased. When the excitation intensity exceeded $70 \mathrm{MW} / \mathrm{cm}^{2}$, which is still about 8 times less than the fabrication laser intensity, we found the spectral narrowing and steep increase in the emission intensity at around $367 \mathrm{~nm}$, although these behaviors were not observed in samples not treated with strong UV irradiation pulses. We found the optimal fabrication laser intensity for fabricating optimal modified GaN surfaces, at $\sim 550 \mathrm{MW} / \mathrm{cm}^{2}$ by varying the fabrication laser intensity. Because the method is quite simple, needing solely the irradiation of intense lasers on a material surface, it may be extended to use with a wide range of other semiconductor materials. Furthermore, the microscale random structures fabricated by this method may find application in photonic materials for use in photo-catalysis, in photoelectric conversion, and in light harvesting.

We thank Professor T. Hashizume and Professor S. Judokazis for their helpful advice. This work was supported by JSPS KAKENHI (17K05016 and 16H06506), the
Dynamic Alliance for Open Innovation Bridging Human, Environment and Materials, the Amada Foundation, and the Nippon Sheet Glass Foundation for Materials Science and Engineering.

${ }^{1}$ H. Amano, K. Hiramatsu, M. Kito, N. Sawaki, and I. Akasaki, "Zn related electroluminescent properties in MOVPE grown GaN," J. Cryst. Growth 93, 79 (1988).

${ }^{2}$ J. Benton, J. Bai, and T. Wang, "Utilisation of GaN and InGaN/GaN with nanoporous structures for water splitting," Appl. Phys. Lett. 105, 223902 (2014).

${ }^{3}$ E. Ogawa and T. Hashizume, "Variation of chemical and photoluminescence properties of Mg-doped GaN caused by high-temperature process," Jpn. J. Appl. Phys. Part 1 50, 021002 (2011).

${ }^{4}$ S. Nakamura, S. Pearton, and G. Fasol, The Blue Laser Diodes (Springer, Berlin, 2000).

${ }^{5}$ S. Nakamura, M. Senoh, S. Nagahama, N. Iwasa, T. Yamada, T. Matsushita, H. Kiyoku, and Y. Sugimoto, "InGaN multi-quantum-wellstructure laser diodes with cleaved mirror cavity facets," Jpn. J. Appl. Phys. Part 1 35, L217-L220 (1996).

${ }^{6}$ E. A. Serban, J. Palisaitis, C. Yeh, H. Hsu, Y. Tsai, H. Kuo, M. Junaid, L. Hultman, P. O. A. Persson, J. Birch, and C. Hsiao, "Selective-area growth of single- crystal wurtzite $\mathrm{GaN}$ nanorods on $\mathrm{SiO}_{\mathrm{x}} / \mathrm{Si}(001)$ substrates by reactive magnetron sputter epitaxy exhibiting single-mode lasing," Sci. Rep. 7, 12701 (2017).

${ }^{7}$ M. Sakai, Y. Inose, K. Ema, T. Ohtsuki, H. Sekiguchi, A. Kikuchi, and K. Kishino, "Random laser action in GaN nanocolumns," Appl. Phys. Lett. 97, 151109 (2010).

${ }^{8}$ H. W. Choi, K. N. Hui, and P. T. Lai, "Lasing in GaN microdisks pivoted on Si," Appl. Phys. Lett. 89, 211101 (2006).

${ }^{9}$ N. M. Lawandy, R. M. Balachandran, A. S. L. Gomes, and E. Sauvain, "Laser action in strongly scattering media," Nature 368, 436-438 (1994).

${ }^{10}$ M. A. Noginov, Solid-State Random Lasers (Springer, New York, 2005).

${ }^{11}$ D. S. Wiersma, "The physics and applications of random lasers," Nat. Phys. 4, 359 (2008).

${ }^{12}$ B. Redding, M. A. Choma, and H. Cao, "Speckle-free laser imaging using random laser illumination," Nat. Photonics 6, 355-359 (2012).

${ }^{13}$ H. Zhu, A. Chen, Y. Wu, X. Ji, Y. He, Z. Qiu, Z. Tang, and S. Yu, "Lowthreshold GaN thin-film random laser through the weak scattering feed back," J. Phys. D: Appl. Phys. 50, 045107 (2017).

${ }^{14}$ C. Cachoncinlle, E. Millom, and A. Petit, "High-resolution emission spectroscopy of random lasing in GaN films pumped by UV-pulsed laser," Opt. Commun. 368, 49-53 (2016).

${ }^{15}$ H. Fujiwara, R. Niyuki, Y. Ishikawa, N. Koshizaki, T. Tsuji, and K. Sasaki, "Low-threshold and quasi-single-mode random laser within a submicrometer-sized $\mathrm{ZnO}$ spherical particle film," Appl. Phys. Lett. 102, 061110 (2013).

${ }^{16}$ T. Nakamura, H. Fujiwara, R. Niyuki, K. Sasaki, Y. Ishikawa, N. Koshizaki, T. Tsuji, and S. Adachi, "Origins of lasing emission in resonance-controlled ZnO random laser,” New J. Phys. 16, 093054 (2014).

${ }^{17}$ H. Fujiwara, T. Suzuki, R. Niyuki, and K. Sasaki, "ZnO nanorod array random lasers fabricated by a laser-induced hydrothermal growth," New J. Phys. 18, 103046 (2016).

${ }^{18}$ R. Niyuki, H. Fujiwara, T. Nakamura, Y. Ishikawa, N. Koshizaki, T. Tsuji, and K. Sasaki, "Double threshold behavior from a resonance-controlled ZnO random laser," APL Photonics 2, 036101 (2017).

${ }^{19}$ C. Xu, J. Zhang, H. Sun, and H. Zhao, "Random lasing emission and oscillation in femtosecond laser machined microstructured $\mathrm{Nd}^{3+}$-doped $(\mathrm{Pb}$, $\mathrm{La})(\mathrm{Zr}, \mathrm{Ti}) \mathrm{O}_{3}(10 / 65 / 35)$ ceramics," RSC Adv. 6, 95442-95448 (2016).

${ }^{20}$ J. Bonse, S. Höhm, S. V. Kirner, A. Rosenfeld, and J. Krüger, "LaserInduced Periodic Surface Structures - A Scientific Evergreen," IEEE J. Sel. Top. Quantum Electron. 23, 9000615 (2017).

${ }^{21}$ L. Wang, B. Xu, X. Cao, Q. Li, W. Tian, Q. Chen, S. Judokazis, and H. Sun, "Competition between subwavelength and deep-subwavelength structures ablated by ultrashort laser pulses," Optica 4, 637-642 (2017).

${ }^{22}$ L. Xu, J. Zhang, H. Zhao, H. Sun, and C. Xu, "Enhanced photoluminescence intensity by modifying the surface nanostructure of $\mathrm{Nd}^{3+}$-doped $(\mathrm{Pb}, \mathrm{La})(\mathrm{Zr}, \mathrm{Ti}) \mathrm{O}_{3}$ ceramics," Opt. Lett. 42, 3303-3306 (2017).

${ }^{23}$ U. Kaufmann, M. Kunzer, H. Obloh, M. Maier, Ch. Manz, A. Ramakrishnan, and B. Santic, "Origin of defect-related photoluminescence bands in doped and nominally undoped GaN," Phys. Rev. B 59, 5561-5567 (1999). 